\section{Welcome to the 13th volume of Future Oncology}
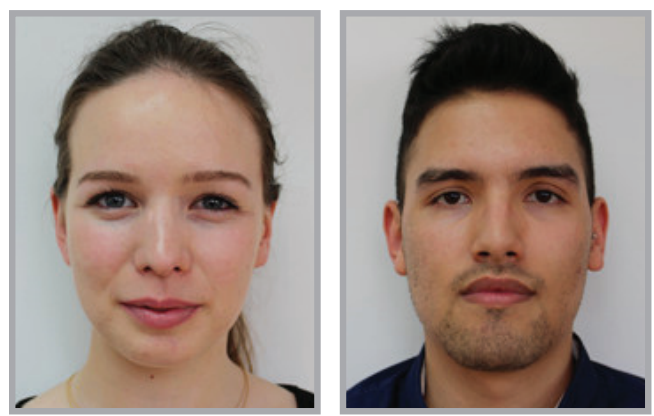

"We look forward to seeing

our readership within the

oncology community grow, and we hope to continue to

circulate our content to an

increasingly global audience.”

Jade Parker ${ }^{*, 1} \&$ Sebastian Dennis-Beron ${ }^{*, 1}$

Accepted for publication: 10 October 2016; Published online: 1 December 2016

To all of our readers, we are delighted to welcome you to the 13th volume of Future Oncology. 2016 was another exciting year for the journal where we developed some exciting changes, and we are proud to present some of our content highlights below. To note, Future Oncology's frequency will now increase from 24 to 30 issues a year in 2017, which allows us to accommodate for the high levels of high-quality manuscripts that out journal continues to regularly receive.

We would also like to take this opportunity to thank all of our valued Editorial Board members, readers and contributors for their continued support; we very much look forward to seeing the journal progress and develop as we move into 2017.

\section{Content highlights of 2016}

At the time of writing, our most read article (October 2016) was a piece by Brahmer, Hammers and Lipson from the Sidney Kimmel Comprehensive Cancer Center at the Johns Hopkins School of Medicine (MD, USA) evaluating nivolumab, a human IgG4 checkpoint inhibitor. This provides an early evaluation of the drug, which has now gone on to be the US FDA approved as an immunotherapy for a series of cancers including squamous and nonsquamous non-small-cell lung cancer, renal cell carcinoma and pivotally melanoma [1].

Another one of our most popular articles was a Clinical Trial Protocol authored by Nowakowski and Vitolo from the Mayo Clinic (MN, USA) that outlines the ongoing ROBUST clinical trial (NCT02285062) that is now in Phase III; the trial assesses the efficacy and safety of combining rituximab with cyclophosphamide, doxorubicin, vincristine and prednisone (R-CHOP) in activated $\mathrm{B}$-cell (ABC)-type diffuse large B-cell lymphoma [2].

Also highly read was another Clinical Trial Protocol, the Phase III HELIOS trial (trial registration: EudraCT No. 2012-000600-15; UTN No. U1111-11353745) by Hallek et al. from the University of Cologne (Germany). The team utilizes the International Workshop on Chronic Lymphocytic Leukemia guidelines to assess whether the addition of ibrutinib to

\section{KEYWORDS}

- cancer • clinical trials

- immunotherapy • oncofertility

- oncology • radiotherapy $\bullet$ research

- translational research

'Future Science Group, Unitec House, 2 Albert Place, London, N31QB, UK

*Author for correspondence: j.parker@futuremedicine.com

**Author for correspondence: s.dennis-beron@futuremedicine.com 


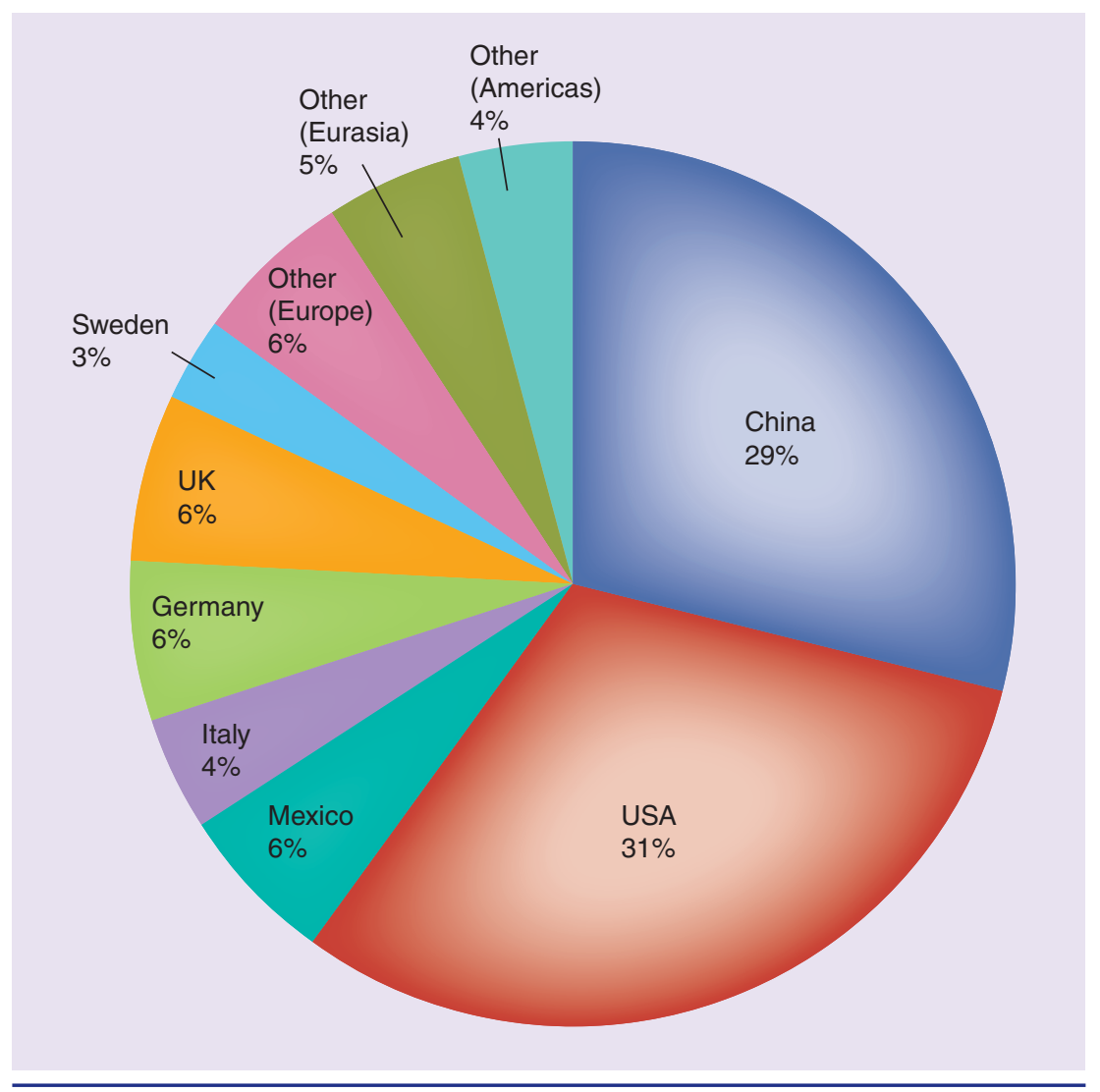

Figure 1. Proportion of readership demographics for Future Oncology in 2016.

bendamustine and rituximab is more efficacious than bendamustine and rituximab in patients with relapsed/refractory chronic lymphocytic leukemia/small lymphocytic lymphoma [3].

A highlight of 2016 was an interview with Patrick Therasse from the European Organisation for Research and Treatment of Cancer (Brussels, Belgium) and Jean-Pierre Abastado from Servier (Slough, UK) discussing the innovations happening at Servier and its research pipeline [4].

Another well-read article was a commentary by Attai et al. from the Collaboration for Outcomes on Social Media in Oncology (COSMO) group (USA) that provides a unique insight into the role, which social media can play in cancer care. Highlights of social media use in oncology include the growth of cancer-specific online Twitter (Inc.) communities [5].

Special mention goes to the following articles, which have achieved the highest Altmetric scores in the journal to date, as we continue our successful partnership with the platform:

- Our highest scores were for a pair of case reports detailing the use of intensity-modulated radiotherapy for mesothelioma and pleuropulmonary synovial sarcoma for the first time 'Intensity-modulated radiotherapy for relapsed malignant pleural mesothelioma' \& 'Role, timing and technique of radiotherapy in pediatric pleuropulmonary synovial sarcoma' by Spatola et al. [6,7] - Altmetric score: 457;

- The previously mentioned 'Social media in cancer care: highlights, challenges and opportunities' by Attai et al. [5] - Altmetric score: 63;

- 'Pixantrone-rituximab versus gemcitabinerituximab in relapsed/refractory aggressive nonHodgkin lymphoma' [8] - Altmetric score: 33;

- We also received a high scores for an article from our Oncofertility special focus issue, 'The challenge of fertility preservation in cancer patients: a special focus issue from Future Oncology' by Grynberg [9] - Altmetric score: 30.

It is interesting to see where the journal's content is having the most impact; in 2016, our journal's content was most read by experts in the USA (31\%) and China (29\%) (Figure 1). Nevertheless, our work reaches across the globe with not only a continued following in the UK and Italy but also emerging readership in Mexico and Eurasia. We look forward to seeing our readership within the oncology community grow, and we hope to continue to circulate our content to an increasingly global audience.

\section{Social media}

Future Oncology is very active across various forms of social media including Linkedin on our Future Science Oncology Group [10] and Twitter, @fsgfon [11]. We regularly post journal highlights including newly published content and the latest news in oncology. We have thoroughly enjoyed engaging with oncology professionals across the field; we continuously strive to grow our network presence and welcome our readers to connect with us on social media.

\section{Conclusion}

We appreciate all feedback from the oncology community regarding the direction of our content such as 'hot topics' in the field and trends that you feel should be covered in the Future Oncology. We welcome unsolicited article proposals and would be delighted to hear from you. In particular, we encourage submissions on novel and translational work such as: 
- Selective and 'personalized' approaches;

- Integration of diagnostic and therapeutic approaches;

- Profiles of new anticancer agents;

- Biological processes involved in cancer and how new understanding will impact treatment;

- Clinical implications and applications for new biomarkers;

- Screening programs and methodology;

- Impact of molecular genetics on prevention, screening, diagnosis and treatment.
We greatly look forward to collaborating with you all over the next year and hope Future Oncology continues to fulfil its role in the medical community and grow and develop as a journal.

\section{Financial \& competing interests disclosure}

$S$ Dennis-Beron and J Parker are employees of Future

Medicine Ltd. The authors have no other relevant affilia-

tions or financial involvement with any organization or entity with a financial interest in or financial conflict with the subject matter or materials discussed in the manuscript apart from those disclosed.

No writing assistance was utilized in the production of this manuscript.

\section{References}

1 Brahmer JR, Hammers H, Lipson EJ. Nivolumab: targeting PD-1 to bolster antitumor immunity. Future Oncol. 11(9), 1307-1326 (2016).

2 Nowakowski GS, Chiappella A, Witzig TE et al. ROBUST: lenalidomide-R-CHOP versus placebo-R-CHOP in previously untreated ABC-type diffuse large B-cell lymphoma. Future Oncol. 12(13), 1553-1563 (2016).

3 Hallek M, Kay NE, Osterborg A et al. The HELIOS trial protocol: a Phase III study of ibrutinib in combination with bendamustine and rituximab in relapsed/refractory chronic lymphocytic leukemia. Future Oncol. 11(1), 51-59 (2015).
4 Therasse P, Abastado JP. Servier's pipeline in oncology: moving from research to patients. Future Oncol. 12(5), 589-594 (2016).

5 Attai DJ, Sedrak MS, Katz MS et al. Social media in cancer care: highlights, challenges and opportunities. Future Oncol. 12(13), 1549-1552 (2016).

6 Spatola C, Militello C, Tocco A et al. Intensity-modulated radiotherapy for relapsed malignant pleural mesothelioma. Future Oncol. 12(Suppl. 23), 67-71 (2016).

7 Spatola C, Tocco A, Milazzotto R et al. Role, timing and technique of radiotherapy in pediatric pleuropulmonary synovial sarcoma. Future Oncol. 12(Suppl. 23), 73-77 (2016).
8 Belada D, Georgiev P, Dakhil S et al. Pixantrone-rituximab versus gemcitabinerituximab in relapsed/refractory aggressive non-Hodgkin lymphoma. Future Oncol. 12(15), 1759-1756 (2016).

9 Grynberg M. The challenge of fertility preservation in cancer patients: a special focus issue from Future Oncology. Future Oncol. 12(14), 1667-1669 (2016).

10 Future Science Oncology LinkedIn Group. LinkedIn (2016).

www.linkedin.com/groups/8234713

11 Future Oncology Twitter Handle. Twitter (2016).

https://twitter.com/fsgfon 